\title{
Alternate Formulations of the Reducibility Problem of Open Shop Sequences Minimizing the Makespan
}

\author{
Tanka Nath Dhamala \\ Central Department of Mathematics, Institute of Science and Technology \\ Tribhuvan University, Kathmandu, Nepal \\ Email: dhamala@yahoo.com
}

\begin{abstract}
The decision problem whether a given open shop sequence, minimizing the maximum completion time, is irreducible has been considered in the last 20 years. The problem has diversified applications in industries and communications. By now, a number of algorithms based on the specific properties of the corresponding sequence graph are proposed. Thus the problem is solved only partially and only in some special cases, but not in general yet. A number of open problems and conjectures carried out in this research have been posed, so far. In this paper, we present a brief sketch of these ideas with different formulations of the reducibility of open shop sequences and expose how important are the roles of conflict resolution reaching a conclusion to its end. Paths on the so-called H-comparability graphs with respect to the implication classes play vital roles in it.
\end{abstract}

Keywords: scheduling, sequencing, open shop, comparability graph, reducibility, complexity.

\section{Introduction}

In an open shop scheduling problem, each job $i \in\{1,2, \ldots, n\}$ has to be processed on each machine $j \in\{1,2, \ldots, m\}$ exactly once without preemption for $p_{i j}>0$. Let be $P=\left[p_{i j}\right]$ (matrix of processing times), $O I J=\left\{o_{i j} \mid p_{i j}>0\right\}$ (set of all operations) and $C=\left[C_{1}, C_{2}, \ldots, C_{n}\right]$ (vector of completion times of all jobs). The objective function is $C_{\max }=\max _{i \in I} C_{i}$. One of the major tasks is to find a feasible (acyclic) combination of all machine orders and job orders, called sequence that minimizes $C_{\max }$, that is an optimal schedule. We denote the set of all $\mathrm{n} \times \mathrm{m}$ sequences by $S I J$. This problem $O / / C_{\max }$ is solvable in time $O(n)$ for $m=2$, and is strongly $N P$-hard for $n \geq 3$, [15].

A sequence $A$ is called reducible to another sequence $B$ if $C_{\max }(B) \leq C_{\max }(A)$ for all $P \in P_{n m}$, we write $B \underline{\square}$. It is called strongly reducible, denoted by $\mathrm{B} \prec \mathrm{A}$, if $B \underline{\square} A$ but not $A \underline{\square} B$, and called similar, denoted by $\mathrm{A} \sim \mathrm{B}$, if both $B \underline{\square} A$ and $A \underline{\square} B$ hold. A sequence $A$ is called irreducible if there exists no other non-similar sequence $B$ to which $\mathrm{A}$ can be reduced. The irreducible elements are the minimal sequences with respect to the partial order $\prec$ and thus they are locally optimal. The set forms a potentially (universally) optimal solution space which contains at least one optimal sequence for arbitrary processing time $p_{i j},[9,22,23]$. This 
dominance relation was introduced in [17]. One of the most important motivations to consider this research is that the number of irreducible sequences is very small in comparison to the number of all sequences, $[22,5]$.

Consider a row permutation $\pi_{r} \in S_{n}$, a column permutation $\pi_{c} \in S_{m}$, a transposition $\Phi \in Z_{2}$, and a reversion $\Psi \in Z_{2}$ of a sequence, where $S_{t}$ denotes the symmetric group of order $t$ and $Z_{2}$ denotes the cyclic group of order two. For two given sequences $A$ and $B$, they are called structure isomorphic, graph isomorphic or permutation isomorphic, denoted by $\cong_{\mathrm{s}}, \cong_{\mathrm{g}}$ or $\cong_{\mathrm{p}}$ if there exists a mapping such that $\left(\pi_{r}, \pi_{c}, \Phi, \Psi\right) A=B,\left(\pi_{r}, \pi_{c}, \Phi\right) A=B$ or $\left(\pi_{r}, \pi_{c}\right) A=B$, respectively.

Each of these isomorphism relations defined above yields an equivalence relation decomposing the sequence space into disjoint isomorphism classes. Two sequences $A$ and $B$ in the same isomorphism class have the invariant property that one is irreducible if and only if the other is also irreducible. However, these structures are not fully understood yet though they play a key role in determining the number of all sequences and the number of irreducible sequences.

Informally, a decision problem is said to be in the class $P$ if there exists a deterministic algorithm which solves the problem in polynomial time. A decision problem is in $N P$ if a positive answer can be verified in polynomial time. That is, there exists a nondeterministic polynomial time algorithm solving it. A decision problem is called $N P$-complete if the problem belongs to $P$, then $N P=P$ holds. For instance, the graph isomorphism problem has been shown to be in $N P$, but not known to be $N P$-complete, neither is it known to be in $P$. However, some special cases are polynomial solvable. The isomorphism of two sequences $A$ and $B$ of the same size $n \times m$ is decidable in $O\left(\min \left\{m n^{2}, m^{2} n\right\}\right)$ time, $[4,10]$. An efficient algorithm to decide whether a given connected digraph is a shop graph or a sequence graph is given in $[10,4,16]$. Both algorithms have time complexity $O\left(\max \left\{m n^{2}, m^{2} n\right\}\right)$. A systematic analysis of the complexity classes can be found in [11].

The considered problem of irreducibility on an operation set with spanning tree structure is polynomially solved, [6]. This concept is generalized by considering a dominance relation between a sequence and a set of sequences, [22]. The $3 \times 3$ open shop problem has been solved and a mixed integer programming has been formulated, [22]. For further extensions we refer to, $[16,23,10]$. Several necessary and sufficient conditions, which can be tested in polynomial time, and some computational results have been proposed, [5]. However, up to now, no polynomial time algorithm is known for the decision whether a sequence is irreducible in general. The problem reducibility is in the class $N P$ and the problem irreducibility is in the class co- $N P$. For a reducible sequence, the reducibility can be proved with nondeterministic polynomial time. We refer to the references, $[9,2,8,1]$, for the updated results.

Recently, two algorithms, one with polynomial time though of high complexity and the other an enumerative, have been proposed which differ in the nature of the diagonal edges on the corresponding H-comparability graph of the given sequence, [2, 1]. They discuss quite meaningfully on the resolutions of the conflicts in this graph. In this paper, we mostly deal with the current issues and sketch the main ideas on how important are the roles of these resolutions reaching a conclusion to its end. 
Section 2 gives the mathematical formulations of the reducibility problem. In Section 3, we give different approaches of the problem formulation. Section 4 summarizes the roles of conflict resolutions for a solution of the formulated problem. The final section concludes the paper.

\section{Basic Concepts}

\subsection{The Model}

A latin rectangle $A[n, m, q]=\left[a_{i j}\right]$ is a matrix of size $n \times m$ with $a_{i j} \in\{1,2, \ldots, q\}$ such that each integer of the symbol set $\{1,2, \ldots, q\}$ occurs at most once in each row and in each column of $A$. If $n=m=q$ holds, then the matrix is a latin square of order $n$, [7]. Here, we use the blockmatrices model [3] that uses special latin rectangles satisfying so-called the sequence property which states that for each integer $a_{i j}>1$ there exists $a_{i j}-1$ in row $i$ or in column $j$ or in both. These matrices are in one-to-one correspondence with the sequence graph that is an acyclic orientation of the disjunctive graph, [21]. Moreover, this mapping is polynomial time transferable.

The $n \times m$ matrices of all job orders and machine orders are denoted by $J O$ and $M O$, respectively. For any pair $(M O, J O)$, we define the shop graph $G_{(M O, J O)}=(O I J, E)$ where the arc set reflects the union of all machine orders and all job orders, [10]. A shop graph is a sequence graph (nonsequence graph) if it is acyclic (cyclic). For each sequence graph $G_{(M O, J O)}$ we can describe the sequence $(M O, J O)$ by a latin rectangle $A=\left[a_{i j}\right]$, where $a_{i j}=\operatorname{rank}\left(o_{i j}\right)$ with the sequence property. Recall that the rank of a vertex $o_{i j}$ is the number of vertices on a longest path from a source to this vertex. An arc from a vertex $o_{i j}$ to another vertex $o_{k l}$ exists if and only if $i=k$ or $j=$ $l$ is satisfied and $a_{i j}<a_{k l}$ holds.

\subsection{Hamming Graph}

Let the Hamming graph $K_{n} \times K_{m}$ be denoted by $G_{O I J}$. Let the transitive orientation of the sequence graph and its symmetric closure, which is a H-comparability graph, be denoted by $A^{t r}$ and $\left[A^{t r}\right]$, respectively. Both can be obtained in polynomial time, [19]. Here, $\left[A^{t r}\right]=\left(\right.$ OIJ, $\left.A^{t r}+\left(A^{t r}\right)^{-1}\right)=$ $\left(O I J, E_{r(A)} \cup E_{d(A)}\right)$ is undirected graph, where $E_{r(A)}$ and $E_{d(A)}$ represent the sets of all regular edges (i.e., all vertical and horizontal edges) and all diagonal edges, respectively, and $\left(A^{t r}\right)^{-1}$ denotes the graph of reverses of edges of the symmetric closure $A^{t r}$.

A graph that has a transitive orientation is called a comparability graph, [12], and it is called prime if it is uniquely orientable. These graphs have interpretations in shop scheduling problems. A Hamming graph which is restricted on a partial operation set defined as

$$
O I J=\left\{o_{i j} o_{k l} \mid p_{i j}>0, I=k \text { or } j=l\right\}
$$

is called H-graph. A comparability graph $G=(V, E)$ with $V=O I J$ which contains an H-graph is called H-comparability graph. Recall that not all Hamming graphs are comparability, for example, $K_{2} \times K_{3}$.

The following comparability graph problem is solvable. Given a graph $G=(V, E)$, is it a comparability graph? There exist various methods to solve this problem. For example, test of the uniqueness of its implication classes or the test of the corresponding bipartite graphs property needs $O(|V||\mathrm{E}|)$ time. Another variant would be the approach of modular decomposition with complexity $\mathrm{O}((|V|+|E|) \log |V|)$. The recent and the best approach is to determine the transitive 
orientation of the comparability graph rather than testing whether an acyclic orientation exists. This requires $\mathrm{O}(|E|+|V|)$ time.

\section{Example 1}

Consider a 3-jobs 4-machines open shop problem with the machine orders

$$
J_{1}: M_{2} \rightarrow M_{4} \rightarrow M_{1}, J_{2}: M_{3} \rightarrow M_{2} \rightarrow M_{4} \rightarrow M_{1}, J_{3}: M_{1} \rightarrow M_{3} \rightarrow M_{4}
$$

and the job orders

$$
\mathrm{M}_{1}: \mathrm{J}_{3} \rightarrow \mathrm{J}_{1} \rightarrow \mathrm{J}_{2}, \mathrm{M}_{2}: \mathrm{J}_{1} \rightarrow \mathrm{J}_{2}, \mathrm{M}_{3}: \mathrm{J}_{2} \rightarrow \mathrm{J}_{3}, \mathrm{M}_{4}: \mathrm{J}_{1} \rightarrow \mathrm{J}_{3} \rightarrow \mathrm{J}_{2} .
$$

Then the corresponding rank matrices are, the machine order matrix $M O=\left(\begin{array}{cccc}3 & 1 & . & 2 \\ 4 & 2 & 1 & 3 \\ 1 & \cdot & 2 & 3\end{array}\right)$,

the job order matrix $J O=\left(\begin{array}{cccc}2 & 1 & \cdot & 1 \\ 3 & 2 & 1 & 3 \\ 1 & . & 2 & 2\end{array}\right)$ and the sequence $B=\left(\begin{array}{llll}3 & 1 & \cdot & 2 \\ 5 & 2 & 1 & 4 \\ 1 & \cdot & 2 & 3\end{array}\right)$.

Given the matrix of processing times $P=\left(\begin{array}{llll}6 & 1 & \cdot & 1 \\ 3 & 6 & 4 & 2 \\ 2 & \cdot & 1 & 3\end{array}\right)$, the matrix of completion times is obtained as $C=\left(\begin{array}{cccc}8 & 1 & . & 2 \\ 15 & 10 & 4 & 12 \\ 2 & . & 5 & 8\end{array}\right)$ which is the schedule corresponding to the sequence $B$ and the matrix $P$ with $C_{\max }=15$. Remark that this value has to be minimized for the optimality.

\subsection{Implication Classes}

Two edges $a b, c d$ in the sequence graph $G_{S}$ are said to be in $\gamma_{S}$-relation, denoted by ab $\gamma_{s}$ cd, if and only if they are in $\gamma$-relation, denoted by ab $\gamma \mathrm{cd}$, in the transitive closure $S^{t r}$. For two edges $a b, c d$ in a graph $G=(V, E)$ a - relation is defined as follows: $\forall a b, c d \in E: a b c d \Leftrightarrow a=c$ $\wedge b d \notin E$, or $b=d \wedge a c \notin E$, or $a b=c d$.

Two regular edges $e, e^{, \in{ }^{E}} r(S)$ are said to be connected by a $\gamma_{S}$-path if there exists a chain of edges $e=e_{0}, e_{1}, \ldots, e_{m}, e_{m+1}=e^{\prime}$ from the set of regular edges $E_{r(S)}$ such that it holds $e \gamma_{\mathrm{S}} e_{1} \gamma_{\mathrm{S}} e_{2}$ $\ldots e_{m} \gamma_{S} e^{\prime}$. This relation defines the transitive closure, denoted by $\gamma_{S}^{\mathrm{tr}}$, of the $\gamma_{S}$ - relation on $E_{r(S)}$. The relation $\gamma_{s}^{\text {tr }}$ partitions the set $E_{r(S)}$ into disjoint equivalent classes, called sequence implication classes, which decompose the set of all edges into disjoint equivalent classes in the comparability graph. The set of all sequence implication classes is denoted by $P_{S} T R=\left\{P_{1}, \ldots, P_{k}, P_{1}^{-1}, \ldots\right.$, $P_{k}^{-1}$. The equivalent relation similarly induced by the $\gamma$-relation also partitions the set of edges $E$ in $S^{t r}$, called the implication classes. The set of all implication classes is denoted by $\mathrm{I}_{[S} T R_{]}=$ $\left\{I_{1}, \ldots, I_{l}, I_{1}^{-1}, \ldots, I_{l}^{-1}\right\}$.

If a sequence implication class contains more than two edges, then at least one vertical edge and at least one horizontal edge must be contained in it. The extended sequence implications are the 
minimal sets containing all transitive edges of the corresponding classes. Each sequence implication class is contained in some implication class.

The sequence implication classes introduced in [23] (see also, [17]) play important roles in the theory of irreducibility in open shop problem. For example, a sequence with only one sequence implication class is irreducible. Therefore, all latin squares are irreducible having only one sequence implication class in each. Furthermore, the sequence implication classes are the basic elements that generate the set of all sequences by their combinations, in particular, the set of all reducible sequences are generated by them, $[2,1]$.

The notion of irreducibility depends on the characteristics of the diagonal edges of the $\mathrm{H}$ comparability graph $\left[A^{t t}\right]=\left(O I J, E_{r(A)} \cup E_{d(A)}\right)$. An edge $\mathrm{e} \in \mathrm{E}_{\mathrm{d}(\mathrm{A})}$ is called stable if it is contained in every irreducible sequence of the sequence $\mathrm{A}$. The set of all stable edges may be empty since two irreducible sequences may not have a common edge which is stable. An edge $e \in E_{d(A)}$ which belongs to an extended sequence implication class is always stable, and therefore we call is by trivial-stable. If all edges in the transitive closure $\left[A^{t r}\right]$ are trivial-stable, then the sequence is irreducible and the problem is thus solved polynomially. Let $e^{\wedge}=\left\{e, e^{-1}\right\}$. A stable edge $e \in E_{d(A)}$ which is not contained in an extended sequence implication class is called nontrivial-stable. If one could prove the stability of an edge in polynomial time, then the problem of irreducibility would be polynomially solvable. If one could find a randomized algorithm to test it, the irreducibility problem would be in $N P \cap c o-N P$.

\subsection{Maximal Paths}

A path $w_{A}$ with vertex set $V\left(w_{A}\right)$ in the sequence graph $G_{A}$ (equivalently, in the sequence $A$ ) is called maximal if there does not exist another path $w_{A}{ }^{*}$ in it with $V\left(w_{A}\right) \subset V\left(w_{A}{ }^{*}\right)$. But, the set of all maximal paths in $G_{A}$, denoted by $W_{A}$, contains an exponential number of maximal paths. For example, there are $n 2^{n-1}$ maximal paths in a latin square of order $n$.

Clearly, the set $W_{A}$ contains the longest path depending on the processing times. A sequence $A$ is reduced to another sequence $B$, if and only if for all $w_{B} \in W_{B}$ there exists $w_{A} \in W_{A}$ with $V\left(w_{B}\right) \subseteq$ $V\left(\mathrm{w}_{\mathrm{A}}\right)$. If $B \prec A$, then there exists $w_{B} \in W_{B}$ with $V\left(w_{B}\right) \subset V\left(w_{A}\right)$ for some $w_{A} \in W_{A}$.

Note that $B \prec A$ does not necessarily imply $C_{\max }(B)<C_{\max }(A)$. It is true if there exists a unique maximal path in $A$. For example, consider $P=\left[p_{i j}\right]$ with $p_{i j} \in Z_{+}$such that $p_{i j}=k^{\prime}$ if $o_{i j} \in V\left(w_{A}\right)$, and 1 , otherwise, where $k^{\prime}>n m$, then $C_{\max }(A)>\mathrm{C}_{\max }(B)$, if $\mathrm{A} \succ \mathrm{B}$ holds.

Lemma $1 \mathrm{~A}$ sequence $A$ is reducible to another sequence $\mathrm{B}$ of the same format if and only if for all maximal paths $w_{B}$ in $B$, there exists a maximal path $w_{A}$ in $A$ such that the inclusion $V\left(w_{B}\right) \subseteq V$ $\left(w_{A}\right)$ is satisfied.

However, the decision whether a given sequence $\mathrm{A}$ is irreducible, or it is reducible or similar to another sequence B simply by using the related definitions (cf. Lemma 1) takes exponential time. Following lemma, [20], is useful in testing these results for given two sequences.

Lemma 2 Let be the operation sets such that $O I J^{\prime} \subseteq O I J$. Then there exists a path $w_{A}$ the closure $A^{t r}$ with $V\left(w_{A}\right)=O I J^{\prime}$ if and if $O I J^{\prime}$ is a clique in $\left[A^{t r}\right]$. Moreover, such a path is unique for the clique $O I J^{\prime}$ in $\left[\mathrm{A}^{\mathrm{t} t}\right]$. 


\section{Alternative Formulations}

Let $A \in S I J$ be an open shop sequence for the open shop scheduling problem $O / / C_{\max }$. We state the following recognization problems on the same operation set $O I J,[1,2,8]$.

Irrededucibility 1 Is the sequence $A$ irreducible?

Reducibility 1 Does there exist a sequence $B \in S I J$ such that $B \prec A$ ?

Reducing 1 Find a sequence $B$, if it exists, such that $B \prec A$.

Clearly, irreducibility and reducibility are complement decision problems, however, reducing is the constructive optimization problem to the decision problem reducibility.

Let $A$ and $B$ be two sequences on the same operation set for the problem $O / / C_{\max }$. Theorem 1 , gives the first polynomial test of irreducibility, reducibility or similarity, for given two sequences.

Theorem 1 The sequence $A$ is reducible, strongly reducible or similar to the sequence $B$ if and only if $\left[B^{t r}\right] \subseteq\left[A^{t t}\right],\left[B^{t r}\right] \subset\left[A^{t t}\right]$ or $\left[B^{t t}\right]=\left[A^{t r}\right]$, respectively.

Proof: Let $A$ and $B$ be two given open shop sequences of the same format. To determine the transitive closures $A^{t r}$ and $B^{t r}$ of the sequence graphs $G_{A}$ and $G_{B}$ and then to test if the symmetric closure $\left[B^{t r}\right]$ is a subgraph of the symmetric closure $\left[A^{t r}\right]$, it takes $O\left(n^{2} m^{2}\right)$ time for the operation set with $|O I J|=n m$. The other implications follow from the definitions and the Lemma 2, see [10] for details.

Theorem 2 The problem reducibility is in $N P$. The problem irreducibility is in co- $N P$.

Proof: The conditions in Theorem 1 can be tested in $O\left(\mathrm{n}^{2} \mathrm{~m}^{2}\right)$ time as testing of subgraphs and the construction of transitive closures can be performed with this time.

Thus for a reducible sequence, the reducibility can be proved with nondeterministic polynomial time. As this proof is constructive, such a procedure answers not only to the reducibility but also to the problem reducing. Furthermore, if there exists a NP-test for irreducibility, then the problem of irreducibility is either polynomially solvable or $N P$-incomplete, as far as $\mathrm{P} \neq N P$ holds.

There exist a number of necessary (sufficient) conditions for irreducibility (reducibility) which can be verified in polynomial time, $[22,5,23,16,2,8]$. One of them concerns the reduction of a sequence through the reversion of an implication class in the transitive closure of a sequence, and therefore has a special meaning. The most fundamental one states that a sequence A whose $\mathrm{H}$ comparability graph $A^{t r}$ is not prime is either reducible or is similar to an irreducible sequence $B$ with $B \neq A$ and $B \neq A^{-1}$, [23].

With this and the notion that the irreducible sequences are the minimal elements of the partial order on H-comparability graph $\left[A^{t r}\right]$ containing H-graph $G_{O I J}$, for given $A \in S I J$ and $\left[A^{t r}\right]$, the problems of irreducibility and reducibility can be reformulated as the question of the existence of an H-comparability graph $G$ with $G_{O I J} \subseteq G \subset\left[\mathrm{A}^{\mathrm{tr}}\right]$. Clearly, answer "no" implies irreducibility and answer "yes" implies reducibility.

The notion is related to the graph sandwich problem. The graph sandwich problem for property $P$ is defined as follows: Given two graphs $G_{1}=\left(V, E_{1}\right)$ and $G=\left(\mathrm{V}, \mathrm{E}_{2}\right)$ such that $E_{1} \subseteq E_{2}$, is there a 
graph $G=(V, E)$ such that $E_{1} \subseteq E \subseteq E_{2}$ which satisfies property $P$ ? However, our problem is related to the following recognization problems.

Comparability-graph-sandwich problem: Given two graphs $G_{1}=\left(V_{1}, E_{1}\right)$ and $G_{2}=\left(V_{2}, E_{2}\right)$ with $V_{1} \subseteq V_{2}$ and $E_{1} \subseteq E_{2}$, does there exist a comparability graph $G$ with $G_{1} \subseteq G \subseteq G_{2}$ ?, [13].

Comparability-graph-deletion problem: Given a graph $G=(V, E)$, does there exist a set $M \subseteq E$ of at most $k$ edges deletion of which yields $G$ a comparability-graph?, [24].

The problems comparability-graph-sandwich and comparability-graph-deletion are $N P$-complete. In our case, $G_{1}=K_{n} \times K_{m}$ and $G_{2}=\left[S^{t r}\right]$ for the irreducibility problem of open shop sequences.

A sequence can be obtained from every transitive orientation of an H-comparability graph. If the H-comparability graph $G$ has a sequence orientation $A^{t r}$, then $G=\left[A^{t r}\right]$ is the H-comparability graph to a sequence $A \in$ SIJ. A transitive orientation $\left.T \in T_{[\mathrm{S}}^{\text {tr }}\right]$ is called a sequence orientation if every diagonal edge in $T$ is transitive. If a transitive orientation $B^{t r}$ of $\left[A^{t r}\right]$ is not a sequence orientation, then some diagonal edges of $\left[A^{t r}\right]$ are not in the orientation $B^{t r}$, and $B \prec A$.

To reduce further the given sequence another possibility is to look after the reversion of an implication class or a combination of them. A special and an easiest method to reverse the sequence implication classes can be examined by deleting a single diagonal edge. Deletion of an edge from a transitive reduction can be done easily. However, if $\left[A^{t r}\right]$ can be transitively oriented such that neither e nor $\mathrm{e}^{-1}$ are transitive edges, then the edge $\mathrm{e}^{\wedge}$ can be deleted and the graph $\left[A^{t r}\right]$ $-e^{\wedge}$ is a comparability graph whose sequence orientation reduces $A$ strongly. As transitive orientation of an H-comparability graph can be found in polynomial time and the number of diagonal edges for an $n \times m$ open shop problem is of order $O\left(n^{2} m^{2}\right)$, it can be tested in polynomial time whether a give sequence can be strongly reduced by deleting a diagonal edge. This concludes the following.

Theorem 3 If there exists $e^{\wedge} \in E_{d(A)}$ in $A^{t r}$ such that $A^{t r}-e^{\wedge}$ is a comparability graph, then every transitive orientation of $A^{t r}-e^{\wedge}$ induces a sequence which strongly reduces the sequence $A$. Moreover, this takes a polynomial time.

Thus a sequence $A \in S I J$ can be strongly reduced to a sequence $\mathrm{B} \in \mathrm{S}_{\text {SIJ }}$ which cannot be further reduced by reversing an arbitrary implication class. This can be done in polynomial time. The Hcomparability graph $B^{t r}$ is then either prime or there exist similar sequences to $B$ other than $B^{-1}$. One may attempt to reduce a sequence by this way which does not yield an answer. If one attempts to reduce a sequence by considering the recombination of all implication classes, this also fails because of two reasons. First is that the size of such combinations is $O\left(2^{k}\right)$ for $k$ implication classes and every edge may represent an implication class in the worst case. In average, this would be an efficient way. But the most problematic second reason expresses the fact that such combinations cannot give the whole set of irreducible sequences, in general.

Note that, not every recombination of the sequence implication classes of a sequence $\mathrm{A}$ is even acyclic and yields a sequence B if it is acyclic. The reversion of only implication classes and their recombination does not generate the sequence space. However, the set of all recombination of the sequence implication classes is sufficient. Therefore, taking the more accurate concept of 
sequence implication classes as basis for the space of all sequences, the problems Irreducibility 1 and Reducibility 1 have been formulated as follows.

Irrededucibility 2 Does every feasible recombination of the sequence implication classes of $A$ produce a sequence $B \sim A$ ?

Reducibility 2 Does there exist a feasible recombination of the sequence implication classes of $A$ where at least one diagonal edge of $A$ is missing.

Having seen that removing of one single edge may not yield a strongly reduced sequence but with more than two edges removed at the same time may yield, a study concentrated on the deletion of more edges has been focused. Therefore, a concept of the deletion of a set of diagonal edges rather than trying to remove a single edge has been introduced, [2] (see also, [8, 1]).

A removable set with respect to a given sequence $\mathrm{A}$ is defined to be a set of undirected diagonal edges $M \subseteq E_{d(A)}$. The removable set $M$ is called feasible if $\left[A^{t r}\right]-M$ is an H-comparability graph, and it is called feasibly extendable if there exists a feasible removable set $M^{*}$ of diagonal edges of $\left[A^{\text {tr }}\right]$ such that $M \subset M^{*}$. The set $M$ is called infeasible if it is not feasibly extendable.

Note that a removable set which is not feasible may not be necessarily infeasible. On the other hand, a removable set can be feasible and, in addition, feasibly extendable, too. With this concept, we reformulate the problems of irreducibility and reducibility as follows.

Irrededucibility 3 Is every removable set $M \subseteq E_{d(A)}$ in $\left[A^{t r}\right]$ infeasible?

Reducibility 3 Does there exist a feasibly extendable removable set $M \subseteq E_{d}(A)$ in $\left[A^{t}\right]$ ?

A normal sequence $A$ has been defined with the property that $\left[A^{t r}\right]$ does not contain any implication class consisting exclusively of diagonal edges, nor any implication class such that the reversion of any implication class (or group of it) reduce it. The former implication class can be deleted and the reduction through the reversion of implication classes can be performed in polynomial time. Therefore, for any sequence $S$ a strongly normal sequence $S$ from $S$ can be determined in polynomial time.

Consider the reversion of only one sequence implication class in a normal sequence $\mathrm{S}$ and consider the recombination of the form $S_{i}=S-P_{i}+P_{i}^{-1}$, from $S=P_{1}+\ldots+P_{k}$ for an arbitrary sequence implication class and test if $S_{i} \prec S$ holds. If it holds we proceed with the sequence $S_{i}$. Unfortunately, such a recombination may not reduce the given sequence at all. Instead, it even may yield an infeasible instance. If one could show that no such examples exist, then we would be able to provide an irreducible sequence from a given normal $\mathrm{S}$ in polynomial time.

\section{Discussion}

Determination of a removable subset $M \subseteq E_{d}(A)$ with given normal sequence A plays an important role in the theory of reducibility of open shop sequences with a aim of minimizing the makespan. 


\subsection{Graph Classes}

Let be a normal sequence. For its reduction by the reversion of a sequence implication class $P_{1}$ against to another sequence implication class $P_{2}$ from the same implication class, all $\gamma$-paths which connect $P_{1}$ and $P_{2}$ have to be cut. But, the output may not yield a comparability graph.

Given $G=(V, E)$, we define the graph $G=(\mathrm{E}, \gamma)$ with an edge $e_{1} e_{2} \in \gamma$ if and only if $e_{1} \gamma e_{2}$ in $G$. For a given $S$, from the $\gamma$-graph $G=\left(E_{r(S)}+E_{d(S)}, \gamma\right)$ with contraction of edges in $\gamma$-relation, we define the factor graph $G_{F}(S)$. The vertex set $G_{F}(V)$ contains an $\operatorname{arc} v \in E_{d}$ or the extended sequence implication classes in $P_{S}$ and $P_{S}-1$. An undirected edge $e_{1} e_{2}$ belongs to $G_{F}(E)$ if and only if there exists a $\gamma$-relation between nodes or set of nodes $e_{1}$ and $e_{2}$.

Every $\gamma$-path in $\left[S^{t r}\right]$ between two classes $P_{1}$ and $P_{2}$ contains at least one diagonal edge. For feasibility of $M$ every such path has to be broken, in order to avoid a connection in $\left[S^{t t}\right]-M$. Not all $\gamma$-paths are destroyed if $\mathrm{P}_{1}$ and $P_{2}$ belong to one connected component of $G_{F}-M$.

The removal of an edge result new $\gamma$-relations. The consequence graph informs which sequence implication classes are merged by the removal of $e^{\hat{i}} \in E_{d(S)}$.

Let $\left(O I J, E_{r(S)}+E_{d(S)}\right)$ with $\left|\mathrm{E}_{\mathrm{d}}\right|=\mathrm{d}$ be the H-comparability graph to the given $S \in S I J$,

$\mathrm{P}_{\mathrm{S}}=\left\{P_{1}, \ldots, P_{k}\right\}$ and $E_{r}=P_{1}+\ldots+P_{k}+P_{1}^{-1}+\ldots+P_{k}^{-1}$. The consequence graph $G_{K}(S)=\left(V_{K}, E_{K}\right)$ is defined as follows. The set of nodes is $V_{K} \subseteq E_{d(S)}+P_{S}+P_{S}-1$. Two edges $e^{\prime}$ and $e^{\prime \prime}$ from $V_{K}$ are connected by an undirected edge of color $i \in\{1, \ldots, d\}$ when the removal of $e_{i} \in E_{d}$ forms a $\gamma$-relation between e' and $e^{\prime \prime}$ or between the sequence implication classes, they represent, respectively. That is, $E_{K}=\left\{e^{\prime} e^{\prime \prime}\right.$ with color $i \mid e^{\prime} \gamma e^{\prime \prime}$ in $\left.\left[S^{t r}\right]-e_{i}, e_{i} \in E_{d}\right\}$.

The set $G_{K i}$ represents the subgraph of $G_{K}$ with i ${ }^{\text {th }}$ color such that $G_{K}=G_{K 1}+\ldots+G_{K d}$.

These graphs inform existence of a transitive orientation of $\left[S^{t r}\right]-M$, if $M$ terns out to be feasible. This can be tested faster. However, if this is not the case, the question remains how a none feasible but feasibly extendable set $M$ can be expanded or to prove that the set $\mathrm{M}$ is not only none feasible but is infeasible.

For a given $M \subseteq E_{d(S)}$, the reduction graph $G_{R M}(S)=\left(V_{R M}, E_{R M}\right)$ is defined by inserting into $G_{F}$ all edges from $\mathrm{G}_{K}$ which are colored from $M$ and deleting the nodes which represent edges in $M$ as $G_{R M}(S)=\left[G_{F}+e_{e \in M} G_{K e}\right]-M$. The reduction graph informs about the deletion of nodes from $G_{F}$ and addition of edges between the remaining nodes in $G_{F}$ which induce a new $\gamma$-relation between sequence implication classes.

With these graphs, we know how to recognize a feasible removable set. But the way to decide which additional diagonal edges should be added to a none feasible but feasibly extendable removable set in order to get a feasible removable set is an additional issue, see Section 4.2.

\subsection{Conflicts}

Let $G_{R M}(\mathrm{~S})=\left(V_{R M}, E_{R M}\right)$ be the reduction graph with respect to $M \subseteq E_{d(S)}$. We call a path a conflict in it if there exists a path $\mathrm{W} \subseteq \mathrm{V}_{\mathrm{RM}}$ from a sequence implication class $P_{i} \in V_{R M}$ to its 
reversion $P_{i}^{-1} \in V_{R M}$. The number $l \geq 0$ of diagonal edges contained in the inclusion minimal path $W$ is called the order of the conflict. The conflict of order $1=0$ is called the direct conflict. A reduction graph is called conflict-free if it contains no conflict.

Every conflict in $G_{R M}$ reflects a $\gamma$-path in $\left[S^{t t}\right]-M$ from an extended sequence implication class $P_{i}$ to its reversion $P_{i}^{-1}$. For $M$, in order to be a feasibly extendable, all these conflicts must be dissolved and every one of these $\gamma$-paths must be broken. Also a path between two edges in a graph will only be destroyed when at least one edge from the -path is removed. The conflict can be dissolved by the removal of arbitrary diagonal edges. Thus an extension of $M$ is equivalent to find a set of diagonal edges in $\left[S^{t \prime}\right]-M$ whose deletion from $\left[S^{t t}\right]-M$ will dissolve all conflicts in $G_{R M}$ simultaneously.

For a none feasible removable set $M,\left[S^{t r}\right]-M$ is not a comparability graph which reduces sequence $S$, and corresponding reduction graph is not conflict free. Then we need to be able to add at least one diagonal edge from every conflict to $\mathrm{M}$ in order to yield a feasibly extended set. The inserted diagonal edges from the conflicts in $G_{R M}$ in $M$ must dissolve these conflicts in every case. This will break the paths in $\left[S^{t r}\right]-M$. But, the insertion of edges in $M$ from $\left[S^{t r}\right]-M$ can induce new $\gamma$-relations and yield new conflicts. Therefore, one has to find a subset of $\left[S^{t \prime}\right]-M$ from the remaining diagonal edges so that the deletion of it dissolves the conflicts all at a time and arises no additional conflicts.

If one deletes a non-trivial stable edge from the conflicts, then it does not result a feasibly extendable $M$. If there is a conflict of order zero, then $\mathrm{M}$ is infeasibly removable. However, if all conflicts of order one are determined, and it is possible to insert all edges from these conflicts to the set $M$, then $M$ is feasibly extendable. One should first test if $e^{*}$ in non-trivial-stable with respect to $M$ who belongs to a conflict of higher order.

A diagonal edge $e \in E_{d}^{t r}(S)$ is called magic-stable with respect to $M$ if it does not lead to a direct conflict in $G_{R M *}$, with $M+e \subseteq M^{*}$, through a series of conflicts of order 1. It has not been found any sequence yet which contains a magic-stable edge. If found, the problem of irreducibility is in $N P$ and if proved none existence, then the irreducibility is polynomially solvable. This is the strongest result obtained in this field, recently, $[2,8]$.

\section{Conclusions}

In this paper, we considered the classical open shop scheduling problem in which the problem of irreducibility has been considered since two decades defining the dominance relation in the space of all sequences. This issue is important as the set of all dominant sequences contains an optimal sequence for arbitrary processing times. The complexity status of this problem is not known yet. A decision may be a mathematical challenge. The problem extended for general objectives and arbitrary numerical input data can have still interesting properties. After summarizing the key issues of its structural properties, we have sketched the recent idea of conflict resolution applied in obtaining the reducing a given sequence or concluding its irreducibility.

In this research field, following questions do have quite important significant.

How can be the results on the maximum completion time objective for other regular objectives or other shop environments, like the job shop, generalized? The answer should be positive. An alternative status of the problem of irreducibility is to decide if a magic-edge exists. If no such 
edges, we conclude with polynomial solvability. If such edges exist, we have concluded the status of the problem. However, it is important to improve the achieved complexity results of irreducibility. On the other hand, a development of neighborhood structure of irreducible sequences is a motivating problem from the beginning of this concept in the open shop.

\section{REFERENCES}

1. Andresen, M and Dhamala, T.N. (2010). New algorithms on the reducibility of open shop sequences minimizing the makespan, submitted to Mathematical Methods of Operations Research

2. Andresen, M. (2009). On the complexity of reducibility problems through H-comparability graphs, PhD thesis, University of Magdeburg, Germany

3. Braesel, H. (1990). Latin rectangles in scheduling theory, Dissertation B, University of Magdeburg, Germany

4. Braesel, H., and Kleinau, M. (1996). New steps in the amazing world of sequences and schedules, Math. Comput. Modeling 43: 195-214

5. Braesel, H., Harborth, M. and Willenius, P. (2001). Isomorphism for digraphs and sequences of shop scheduling problems. Journal of Combinatorial Mathematics and Combinatorial Computing 37: $115-128$

6. Braesel, H., Harborth, M. Tautenhahn, T. and Willenius, P. (1999). On the set of solutions of an open shop problem, Annals of Operations Research 92: 241-263

7. Denes, J. and Keedwell, A.D. (1991). Latin squares: new developments in the theory and applications, Annals of Discrete Mathematics

8. Dhamala, T. N. (2002). Shop scheduling solution spaces with algebraic characterizations, $\mathrm{PhD}$ thesis, University of Magdeburg, Germany

9. Dhamala, T. N. (2007). On the potentially optimal solutions of classical shop scheduling problems, International Journal of Operations Research 4: 1-10

10. Dhamala, T.N. (2010). Reducibility problems of open shop sequences minimizing the makespan, Proceedings of the 19th Workshop on Discrete Optimization, Holzhau, Germany, 57-60

11. Garey, M.R. and Johnson, D.S. (1979). Computers and intractability: a guide to the theory of NPcompleteness, W.H. Freeman \& Co., New York

12. Golumbic, M. C. (1977). Comparability graphs and a new matroid, Journal of Combinatorial Theory, Series B 22: 68-90

13. Golumbic, M. C. (2004). Algorithmic graph theory and perfect graphs, 2nd ed. Annals of Discrete Mathematics 57, Elsevier, 2004

14. Golumbic, M. C., Kaplan, H. and Shamir, R. (1995). Graph sandwich problems. J. Algorithms 19: $449-473$

15. Gonzalez, T. and Sahni S. (1976). Open shop scheduling to minimize finish time, Journal of Assoc. Comp. Mach. 23: 665-679

16. Harborth, M. (1999). Structural investigation of shop scheduling problems: number problems, potential optimality and new enumeration algorithms, $\mathrm{PhD}$ thesis, University of Magdeburg, Germany

17. Kleinau, M. (1993). On the structure of shop scheduling problems: number problems, reducibility and complexity, $\mathrm{PhD}$ thesis, University of Magdeburg, Germany 
18. LiSA - A Library of Scheduling Algorithms; http://lisa.math.uni-magdeburg.de/

19. McConnell, R. M. and Spinrad, J.P. (1999). Modular decomposition and transitive orientation, Discrete Mathematics 201: 189-241

20. Redei, L. (1934). Ein Kombinatorischer Satz, Acta, Litt. Sci. Szeged 7: 39-43

21. Sussman, B. (1972). Scheduling problems with interval disjunctions, Z. Operations Research 16: 165-178

22. Tautenhahn, T. and Willenius, P. (2000). How many sequences solve an open shop problem? Preprint, University of Magdeburg, Germany

23. Willenius, P. (2000). Irreducibility theory in scheduling theory, $\mathrm{PhD}$ thesis, University of Magdeburg, Germany

24. Yannakakis, M. (1981). Edge deletion problems. SIAM Journal of Computing 10: 297-309 